\title{
PERCEPÇÃO DO AMBIENTE CONSTRUÍDO E O USO DE TECNOLOGIAS EMERGENTES
}

\author{
PAIVA, Marie Monique Bruère \\ Universidade Federal de Pernambuco, Doutora \\ e-mail: mariem.paiva@gmail.com
}

\begin{abstract}
RESUMO
Entender o ambiente físico a partir da ótica de seu usuário é fundamental para espaços mais adequados às necessidades das pessoas, considerando os ambientes não só pelas características físico-formais, mas também por seus efeitos produzidos nos indivíduos. Assim, o processo de percepção, dinâmico e multissensorial diante da interação homem-ambiente, ocorre através de uma fase de ordem objetiva e de outra de ordem subjetiva. Com o uso de tecnologias emergentes novos direcionamentos vão surgindo, vislumbrando grande potencial para as investigações do ambiente construído, permitindo concepções mais ergonomicamente adequadas.
\end{abstract}

Palavras-chave: Ergonomia do Ambiente Construído; Realidade Virtual; Eletroencefalografia.

\begin{abstract}
Understanding the physical environment from the perspective of its user is fundamental for spaces more suited to people's needs, considering the environments not only for the physical-formal characteristics, but also for their effects produced in the individuals. Thus, the process of perception, dynamic and multisensory in the face of human-environment interaction, occurs through a phase of objective order and another of subjective order. With the use of emerging technologies new directions are arising, glimpsing great potential for investigations of the built environment, allowing more ergonomically appropriate conceptions.
\end{abstract}

Keywords: Ergonomics of the Built Environment; Virtual Reality; Electroencephalography.

\section{INTRODUÇÃO}

O desenvolvimento de ambientes ergonomicamente adequados perpassa por conceitos multidisciplinares das ciências humanas e ciências sociais aplicadas, indo além das dimensões físicas, cultural e de percepção individual (LAKI; LIPAI, 2007). Desse modo, o ambiente construído passa a ser um instrumento no processo de experiências a partir dos canais sensoriais e dos aspectos culturais, onde são criadas as relações afetivas do usuário com os espaços em que ele interage (TUAN, 2013).

Como campo plural e complexo, a prática científica do design tem experimentado novos processos visando à melhoria da qualidade de vida, do conforto e da segurança de usuários. Juntamente com a proliferação do emprego das tecnologias, a Neurociência desponta como suporte eficiente para o reconhecimento de respostas humanas para produtos e ambientes. 


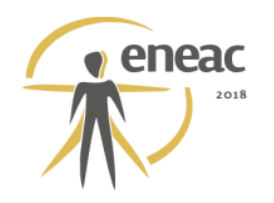

Paralelamente, os avanços tecnológicos têm permitido investigações do ambiente construído com a utilização da Realidade Virtual (PALS et al., 2014; HEYDARIAN et al., 2015) em fases de concepções projetuais, possibilitando correções de inadequações antes mesmo da materialização dos espaços (JOHNSON, 2009).

E é nesse cenário que se insere a pesquisa de doutorado da presente autora. Nela, foram inicialmente avaliadas as preferências de idosos relacionadas a ambientes de salas residenciais através da técnica de Seleção Visual; em uma segunda etapa, foram reconhecidos, em ambientes simulados em Realidade Virtual imersiva, os atributos mais desejados pelos usuários pesquisados. A avaliação perceptiva dos espaços foi suportada por Eletroencefalografia (EEG), com leitura dos biomarcadores de valência emocional, de memória e de atenção. O uso do EEG teve por objetivo identificar emoções (positivas ou não) dos idosos, provocadas por imagens simuladas em 3D com as preferências ambientais identificadas, e compará-las às verbalizações dos idosos durante a visualização dessas imagens. Os resultados dos sinais elétricos cerebrais se mostraram eficientes na identificação de emoções decorrentes dos estímulos visuais dinâmicos apresentados. Dessa forma, revelou-se o caráter inovador e o grande potencial da associação do EEG à Realidade Virtual para as investigações do ambiente construído, permitindo concepções mais ergonomicamente adequadas.

Assim, a seguir, apresenta-se uma breve reflexão sobre a Percepção e as Tecnologias Emergentes, empregadas no estudo realizado - Realidade Virtual e Eletroencefalografia.

\section{PERCEPÇÃO E ERGONOMIA DO AMBIENTE CONSTRUÍDO}

Segundo Vaughn (2015) os efeitos de um ambiente esteticamente agradável são determinantes na condição de bem-estar psicológico das pessoas e na promoção de espaços confortáveis de vivência.

Desse modo, a preocupação com a percepção e comportamento do usuário na promoção de espaços adequados ergonomicamente, e que suscitem a qualidade de vida, tem sido objeto de estudos já consolidado por pesquisas relacionadas ao ambiente e comportamento (RHEINGANTZ, 1995) e à Percepção Ambiental (del RIO, 1999).

Nessa direção, o complexo processo de percepção abrange variáveis de sensação, cognição e avaliação, que acontece mediante uma fase objetiva e outra subjetiva. Johnson (2009) coloca que a percepção atua como a materialização do que se vê; não a imagem exata como se enxerga, mas sob a forma construída de acordo com o repertório de cada indivíduo.

Considerada como um processo, a percepção visual se dá primeiramente pela apreensão da atenção do usuário, surgindo depois o processo de análise e leitura dos detalhes (BAXTER, 2011). Para Löbach (2001), a visão capta a imagem percebida, contudo a decodificação ocorre segundo o interesse, e necessidade (del RIO, 1999) de cada indivíduo, de acordo com seu repertório, atribuindo significados e concretizando a imagem dentro dos limites cognitivos individuais (JOHNSON, 2009).

Por outro lado, Nasar (2008) explica que o processo de percepção envolve o reconhecimento das propriedades físicas do ambiente (forma, tamanho, profundidade, iluminação, etc), juntamente com o estado emocional (ocorrência de natureza racional) e afetivo (ocorrência de natureza inconsciente) do observador. Para as três visões, 0 processo perceptivo culmina por atuar sobre o comportamento humano. 


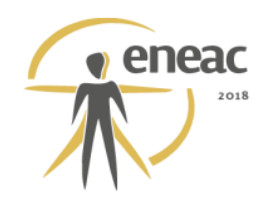

\section{ERGONOMIA E TECNOLOGIAS EMERGENTES}

Toda experiência humana é resultante de atividades cerebrais e da percepção humana (OLIVEIRA, 2012). Assim, as atividades humanas desenvolvidas em espaços físicos são mediadas pela cognição e pela percepção humana, em reação a esses ambientes, no sentido de otimizar as necessidades físicas, psicológicas e emocionais requeridas pelos usuários para esse ambiente.

Para Zeisel (2006) os conceitos e técnicas da Neurociência agregam benefícios à pesquisa emergente (PARASURAMAN, 2012) com abordagem para o sistema ambientecomportamento. Nessa abordagem as atividades repercutem no cérebro, bem como na administração de recepção de informações de conhecimentos ambientais. Assim, o cérebro humano forma mapas que descrevem as relações humanas com os objetos e com os sentimentos (DAMÁSIO, 2000) por meio da consciência ao interagir sensorialmente com os objetos a partir da apropriação das imagens experienciadas (EBERHARD, 2009).

Além do domínio do ambiente construído muitas são as áreas onde a Neurociência pode atuar, tais como, a aviação, a Neuroengenharia, a Genética molecular, a Biotecnologia, a Nanotecnologia e a Realidade Virtual.

\subsection{Realidade Virtual}

Os sistemas de Realidade Virtual se apoiam nos princípios da imersão, interação e envolvimento, por meio da integração de interface ao corpo ou ao ambiente em que está o usuário (HANNS, 2007) e seu feedback sensorial (visual, auditivo e táctil).

Dessa maneira, o nível de imersão em ambientes virtuais é função da associação de elementos, tais como nível de interatividade do usuário com a cena, a complexidade dessa cena e a visão estereoscópica (LÁNYI, 2012). Segundo Tori e Kirner (2006), os sistemas são classificados em ambientes imersivos (onde o usuário encontra-se totalmente inserido no ambiente virtual) e não-imersivos (o usuário tem a presença física no ambiente real, contudo the é proporcionada uma presença parcial no ambiente virtual por meio de um monitor de computador ou projeção).

Como exemplos de ambientes imersivos se destacam os dispositivos do tipo Head-Mounted, Shutterglasses ou em display com múltiplas projeções em paredes - CAVE (Cave Automatic Virtual Environment), exigindo alta performance da computação para um nível de excelência quanto ao realismo do ambiente. Já os ambientes não-imersivos utilizam as mesmas técnicas dos sistemas imersivos, porém empregam hardwares mais genéricos. Nesse sistema, o usuário tem a presença física no ambiente real, contudo lhe é proporcionada uma presença parcial no ambiente virtual.

O uso de tecnologias tem auxiliado as pessoas nas mais diversas áreas, assegurando ganhos de grandes proporções, principalmente no campo da reabilitação, do lazer e também da inclusão social.

\subsection{Eletroencefalografia (EEG)}

O interesse pela investigação de respostas ao comportamento e pensamento humano aplicado à pesquisa tem sido investigado e difundido, sobretudo na área multidisciplinar, envolvendo áreas de conhecimento como a Psicologia Cognitiva, Neurociências, Ciências Cognitivas, entre outras. Relativamente à área da Neurociência, juntamente com a tecnologia e técnicas de EEG, tem feito grandes avanços no reconhecimento do 


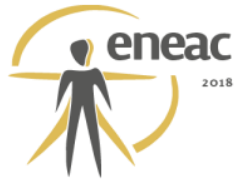

funcionamento do cérebro voltado aos processos cognitivos (SPÍNDOLA, 2010). Também foram evidenciados estudos relacionados à carga mental e de trabalho, e do engajamento do usuário no desenvolvimento da tarefa (VASCONCELOS, 2014).

O EEG se constitui em um procedimento não-invasivo do cérebro para investigação de causas variadas, mas frequentemente está associado à área de saúde, seja para diagnóstico e/ou para reabilitação. Composto por um conjunto de sinais, o EEG reflete a ativação da musculatura da cabeça, os movimentos oculares, bem como a interferência de dispositivos elétricos próximos e a mudança de condutividade nos eletrodos, em função dos movimentos do sujeito examinado, e até mesmo as reações físico-químicas nos locais dos eletrodos.

$\mathrm{Na}$ busca do entendimento mais acurado na interação do usuário com seu meio ambiente (entendendo aqui ser a reunião de espaço e artefatos que concorrem para a realização de qualquer atividade), e devido ao custo acessível do EEG, atualmente o mercado disponibiliza uma variedade de modelos de equipamentos que são utilizados em vários contextos de pesquisas.

\section{CONCLUSÃO}

A Realidade Virtual faz uso dos canais sensoriais humanos, contudo o canal da visão é o mais requisitado. Nessa perspectiva, a simulação de ambientes permite reconhecer situações indesejáveis ainda em fase de planejamento e de projetação dos espaços físicos, sendo tanto mais eficiente quanto for o nível de realismo desses ambientes simulados.

O ambiente construído, seja natural o edificado, envolve todas as ações humanas, e desse modo, a Ergonomia preocupa-se com a maneira como os usuários interagem com esse ambiente, com vistas à adequação espacial e, por conseguinte, da satisfação e da melhoria da qualidade de vida.

As tecnologias emergentes têm evoluído rapidamente com dispositivos atrativos e com utilizações variadas. Nessa direção, o conhecimento das respostas humanas insere diretamente o usuário no contexto participativo da criação, proporcionando produtos e ambientes mais adequados.

Desse modo, a Neurociência aliada à Ergonomia se tornou uma ferramenta potencial para o conhecimento dos processos cognitivos e aprimoramento dos sistemas, onde o homem é o elemento central, conferindo o conforto, a segurança e a qualidade de vida no desempenho das atividades.

\section{REFERÊNCIAS BIBLIOGRÁFICAS}

BAXTER, Mike; tradução Itiro lida. Projeto de produto: Guia pratico para desenvolvimento de novos produtos. 3. Ed. São Paulo: Blucher, 2011.

DAMÁSIO António. The Feeling of What Happens: Body, Emotion and the Making of Consciousness. London: Vintage Books; New Ed edition, 2000.

Del RIO, Vicente. Cidade da Mente, Cidade Real: Percepção Ambiental e Revitalização na Área Portuária do RJ. In: del RIO, Vicente; OLIVEIRA, Lívia (orgs). Percepção ambiental: A experiência brasileira. $2^{a}$ ed. São Paulo: Studio Nobel, pp 3-22, 1999.

EBERHARD, John Paul. Brain landscape: the coexistence of neuroscience and architecture New York: Oxford University Press, Inc., 2009. 


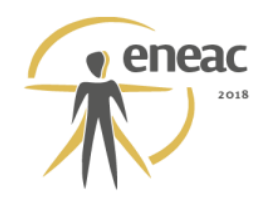

HANNS, Daniela K. Estratégias de imersão: O corpo como interface. In: TORI, Romero; KIRNER, Cláudio; SISCOUTO, Robson. Fundamentos e tecnologia de Realidade Virtual e Realidade Aumentada. Editora: SBC - Sociedade Brasileira de Computação: Porto Alegre, pp 284-287, 2007.

HEYDARIAN, A.; CARNEIRO, J. P.; GERBER, D.; et al. Immersive virtual environments versus physical built environments: A benchmarking study for building design and user-built environment explorations. Automation in Construction, v. 54, pp. 116-126, 2015.

JOHNSON, Angie. Visualisation techniques, human perception and the built environment. Northumbria Working Paper Series: Interdisciplinary Studies in the Built and Virtual Environment, 2 (2), 2009, pp. 93-103.

LAKI , Raquel C.; LIPAI, Alexandre E. Percepção e uso do espaço em arquitetura e urbanismo: um ensaio no ambiente construído. Iniciação Científica, Revista Eletrônica. Ano 1, no 1, p.17-30, 2007.

LÁNYI, Cecília S. Virtual reality and environments. Rijeka, Croatia: In Tech, 2012.

LÖBACH, Bernd. Design industrial - Bases para a configuração dos produtos industriais. $1^{\text {a }}$ edição. São Paulo: Editora Edgar Blücher, 2001.

NASAR, Jack L. Visual Quality by Design. Holland MI: American Society of Interior Designers, Haworth Inc. United States of America, 2008.

OLIVEIRA, Ana Beatriz A. Luz - Elo entre Neurociência e Arquitetura. IPOG Revista Especialize on line. Edição no-3, Maio 2012.

PALS, R.; STEG, L.; DONTJE, J.; et al. Physical features, coherence and positive outcomes of person-environment interactions: a virtual reality study. Journal of Environmental Psychology, 40, 2014, p. 108-116.

PARASURAMAN, R.; CHRISTENSEN, J. \& GRAFTON, S. Neuroergonomics: The brain in action and at work. Neurolmage. Volume 59, 2012, p. 1-3.

RHEINGANTZ, P. A. Centro Empresarial Internacional Rio: análise pós-ocupação por observação participante com ênfase no conforto ambiental. Dissertação (Mestrado em Arquitetura). Rio de Janeiro: FAU UFRJ, 1995.

SPÍNDOLA, Marilda Machado. Habilidade cognitiva especial: medida com eletroencefalografia. Tese (Doutorado em Informática na Educação). Universidade Federal do Rio Grande do Sul, Porto Alegre, 2010.

TORI, Romero; KIRNER, Cláudio. Fundamentos de Realidade Virtual. In: TORI, Romero; KIRNER, Cláudio; SISCOUTTO, Robson. Fundamentos e Tecnologia de Realidade Virtual e Aumentada. Editora SBC - Sociedade Brasileira de Computação: Porto Alegre, pp. 2-21, 2006.

TUAN, Yi-Fu. Espaço e lugar: a perspectiva da experiência. Tradução: Lívia de Oliveira. Londrina: Eduel, 2013.

VASCONCELOS, Christianne S. F. A usabilidade e as tecnologias emergentes no desenvolvimento de produtos de consumo: uma abordagem em ambientes virtuais e neurociência. Tese (Doutorado em Design). Universidade Federal de Pernambuco, Recife, 2014.

VAUGHN, Adel C. Aesthetics and Performance Evaluation of Post-Industrial Public Parks. Landscape Architecture Undergraduate Honors Theses. 6, 2015.

ZEISEL, John. Inquiry by design: environment/behavior/neuroscience in archtecture, interiors, landscape, and planning. New York: W. W. Norton; Revised edition, 2006. 